\title{
Caracterización de la nefritis tubulointersticial aguda en un centro de Cuba, 1988-2018
}

\author{
Raymed A. Bacallao-Méndez ${ }^{1 *}$, Laura López-Marín², Betsy Llerena-Ferrer ${ }^{3}$, Aymara Badell-Moore³, \\ Francisco Gutiérrez-García ${ }^{1}$ y Daniel E. Domínguez-Aguilar ${ }^{1}$
}

${ }^{1}$ Departamento de Docencia e Investigaciones; ${ }^{2}$ Departamento de Patología; ${ }^{3}$ Departamento de Clínica Nefrológica. Instituto de Nefrología Dr. Abelardo Buch López, La Habana, Cuba

\section{Resumen}

Objetivos: Conocer la frecuencia de presentación de las nefritis tubulointersticial aguda (NTIA) en las últimas tres décadas, sus formas de presentación, etiología, manifestaciones clínicas y hallazgos en el sedimento urinario. Material y métodos: Estudio descriptivo, de corte retrospectivo, desarrollado en pacientes cubanos a quienes se les practicó una biopsia renal de riñones propios en el Instituto de Nefrología, La Habana, Cuba, en el periodo de 1988-2018. La información fue procesada de forma automatizada (SPSS 22.0). Se utilizó análisis de distribución de frecuencias, promedio y el test de homogeneidad. Resultados: De 2,472 biopsias practicadas, 77 (3.1\%) fueron diagnosticadas como NTIA. La frecuencia de este diagnóstico se incrementó, desde el 1,1\% en 1988-1998 al 3\% en 1999-2008, y hasta el 4,5\% en 2009-2018 ( $p=0.044)$. La principal forma de presentación fue la injuria renal aguda (IRA) (83,1\%), la tríada de rash cutáneo, eosinofilia y fiebre estuvo presente en el $11.7 \%$, y la causa más frecuente fue la medicamentosa (79.2\%), especialmente por antimicrobianos. Conclusiones: La NTIA se ha incrementado durante el transcurso de los años, y entre los pacientes con IRA, que es su forma de presentación más usual. Las manifestaciones de respuesta de hipersensibilidad no son comunes. Los medicamentos, especialmente los antimicrobianos, son su causa principal.

Palabras clave: Nefritis intersticial aguda. Injuria renal aguda. Biopsia renal.

\section{Characterization of acute tubulointerstitial nephritis in a Cuban center, 1988-2018}

\section{Abstract}

Objectives: To know the frequency of presentation of acute tubulointerstitial nephritis (ATIN) in the last three decades, its forms of presentation, etiology, clinical manifestations and findings in the urinary sediment. Material and methods: $A$ descriptive, retrospective cross-sectional study was carried out in Cuban patients who underwent kidney biopsy of own kidneys at the Nephrology Institute, Havana, Cuba, in the period 1988-2018. The information was processed in an automated way (SPSS 22.0). Analysis of frequency distribution, average and the homogeneity test were used. Results: Of 2472 biopsies performed, 77 (3.1\%) were diagnosed as ATIN. The frequency of this diagnosis increased, from $1.1 \%$ in the period $1988-1998$, to $3 \%$ in $1999-2008$, up to $4.5 \%$ in the period 2009-2018 ( $p=0.044)$. The main form of presentation was acute kidney injury $(A K I)$ (83.1\%), skin rash, eosinophilia and fever triad was present in $11.7 \%$ of patients and the most frequent cause was medication

\section{Correspondencia:}

*Raymed A. Bacallao-Méndez E-mail: raymed@infomed.sld.cu
Fecha de recepción: 15-07-2019

Fecha de aceptación: 30-10-2019 DOI: 10.24875/NEFRO. 19000080
Disponible en internet: 18-12-2019

Nefro Latinoam. 2019;16:39-45 www.nefrologialatinoamericana.com 2444-9032/○ 2019 Sociedad Latinoamericana de Nefrología e Hipertensión. Publicado por Permanyer. Éste es un artículo open access bajo la licencia CC BY-NC-ND (http://creativecommons.org/licenses/by-nc-nd/4.0/) 
Nefro Latinoam. 2019;16

(79.2\%), especially due to antimicrobials. Conclusions: ATIN is an entity that has increased over the years, and among patients with AKI, which is the most common form of presentation. Hypersensitivity response manifestations are not common. Medications, especially antimicrobials, are its main cause.

Key words: Acute interstitial nephritis. Acute kidney injury. Renal biopsy.

\section{Introducción}

La nefritis tubulointersticial aguda (NTIA) se caracteriza histológicamente por la presencia de un infiltrado inflamatorio en el tubulointersticio renal y, en el orden clínico, por grados variables de deterioro de la función renal'.

En varios estudios se ha encontrado que su frecuencia de presentación es de entre el 1-3\% de todas las biopsias renales ${ }^{2,3}$. Sin embargo, en los últimos tiempos se ha reportado un incremento en su incidencia, especialmente en pacientes ancianos ${ }^{4,5}$. No obstante, se considera que es una entidad subdiagnosticada, pues se suele tener un bajo índice de sospecha, y resulta común que a muchos pacientes en quienes se sospecha clínicamente no se les practique una biopsia renal y se utilice tratamiento empírico, a lo que se suma que las formas leves (disfunción renal ligera) pueden no ser detectadas, bien sea por la vaguedad de los síntomas, o porque se atribuyan a otras causas de injuria renal aguda (IRA) ${ }^{6}$.

Para el diagnóstico debe tenerse presente que las NTIA habitualmente se presentan como IRA, aunque pueden tener otras formas de presentación renal ${ }^{7}$. Sin embargo, el diagnóstico puede hacerse difícil, pues la presentación con la tríada clásica de respuesta de hipersensibilidad dada por fiebre, rash cutáneo y eosinofilia es poco frecuente ${ }^{8}$. Del mismo modo, la eosinofiluria (eosinófilos $>1 \%$ de las células blancas en orina), que ha sido considerada un marcador de NTIA, en estudios recientes no ha mostrado una alta sensibilidad y especificidad diagnóstica ${ }^{9}$. El examen del sedimento urinario tampoco resulta muy valioso, pues si bien la leucocituria y los cilindros leucocitarios son comunes, la hematuria, en ocasiones acompañada de cilindros hemáticos, no es rara, a lo que se añade que algunas veces el sedimento resulta pálido ${ }^{10}$.

La proteinuria no suele sobrepasar el valor de $1.5 \mathrm{~g} / \mathrm{día}$, aunque en algunas oportunidades puede alcanzar el rango nefrótico ${ }^{11}$.

Las causas de NTIA son disímiles, los reportes iniciales señalaban a los antimicrobianos betalactámicos, especialmente meticilina, como responsables de la mayoría de los casos, pero en los reportes más recientes, el número de medicamentos, así como las infecciones y otras enfermedades que causan NTIA, se ha incrementado notoriamente ${ }^{9}$. Los estudios actuales identifican los medicamentos como responsables del 70 a $75 \%$ de los casos; las infecciones, del 4 al 10\%; el síndrome de nefritis tubulointersticial y uveítis (TINU, por las siglas de tubulointerstitial nephritis and uveitis), del 5 al 10\%, y las enfermedades sistémicas, como el lupus eritematoso sistémico (LES), síndrome de Sjögren, sarcoidosis, entre otras, del 10 al $20 \%$, aunque las diferencias entre los reportes son importantes ${ }^{5,9}$.

En Cuba y en la región del Caribe no se dispone de datos concernientes a la epidemiología, etiología y a los elementos diagnósticos de las NTIA. A ello se suma que los reportes en el ámbito latinoamericano resultan escasos ${ }^{12}$.

Con el fin de conocer la frecuencia de presentación de las NTIA en las últimas tres décadas, sus formas de presentación, etiología, manifestaciones clínicas y hallazgos en el sedimento urinario, se desarrolla el presente estudio.

\section{Material y métodos}

Se desarrolló un estudio observacional descriptivo retrospectivo de corte transversal en el que se incluyeron todos los pacientes cubanos a los que se practicó una biopsia renal de riñones propios en el Instituto de Nefrología Dr. Abelardo Buch López, La Habana, Cuba (centro de atención terciaria), con diagnóstico de NTIA, en el periodo comprendido entre enero de 1988 y diciembre de 2018. De cada paciente se recogió: fecha de la biopsia renal, edad, sexo, forma de presentación (indicación de biopsia), patrón histológico (difuso o granulomatoso), creatinina sérica, proteinuria de 24 horas, hallazgos en el sedimento urinario, manifestaciones clínicas y etiología, a partir de las boletas de biopsia renal.

Como formas de presentación se consideraron: IRA (según definición de $A K I N)^{13}$, síndrome nefrítico (hematuria más HTA más creatinina $>1.5 \mathrm{mg} / \mathrm{dl}$ ), alteraciones urinarias asintomáticas (presencia de proteinuria $<3.5 \mathrm{~g} / \mathrm{dí} / 1.73 \mathrm{~m}^{2}$ y/o hematuria de más de 30,000 hematíes $/ \mathrm{mm}^{3}$ en la cituria) sin síntomas clínicos, hematuria (macroscópica) y síndrome nefrótico (proteinuria $\geq 3.5 \mathrm{~g} /$ día y albuminemia $<30 \mathrm{~g} / \mathrm{l}$ ). 


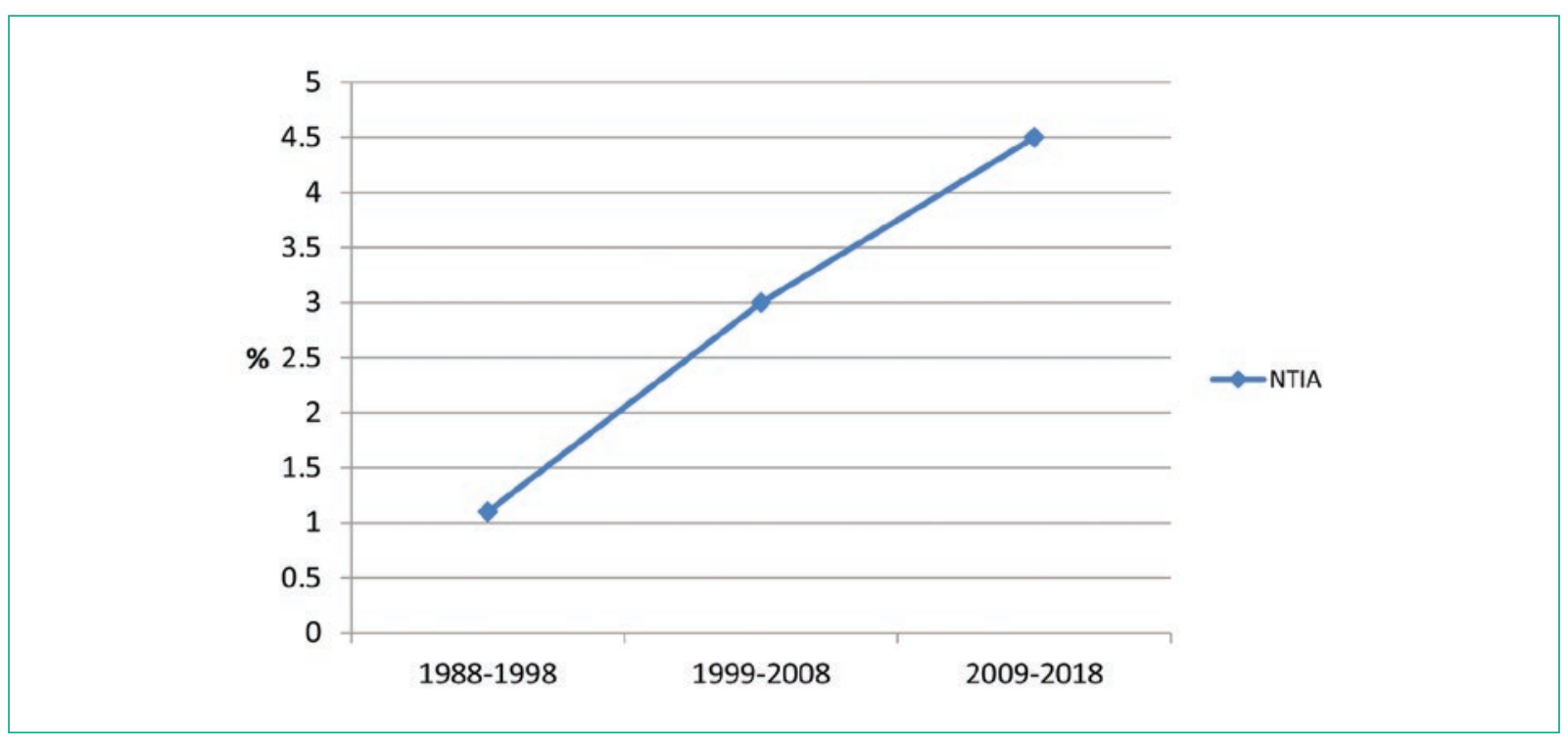

Figura 1. Diagnóstico de NTIA por periodos de estudio.

NTIA: nefritis tubulointersticial aguda.

Se consideró la presencia de eosinofilia cuando el conteo de eosinófilos fue $\geq 500$ eosinófilos/ $\mu$ y leucocituria cuando en el sedimento urinario (por cituria) se hallaron más de 30,000 leucocitos $/ \mathrm{mm}^{3}$.

Las biopsias se hicieron siguiendo el protocolo del servicio. A todas las biopsias se les hizo tinciones de hematoxilina-eosina, tricrómica de Masson, plata metenamina de Jones, ácido periódico de Schiff y rojo Congo. En caso necesario se realizaron otras tinciones especiales. A partir de 2008, a todas las biopsias se les hizo estudio por inmunofluorescencia; se emplearon los antisueros $\lg \mathrm{A}, \lg \mathrm{G}, \lg \mathrm{M}, \mathrm{C} 3, \mathrm{C} 1 \mathrm{q}$, fibrinógeno, kappa y lambda. Además se realizó determinación de IgG4 por técnica inmunohistoquímica.

Se consideró la existencia de NTIA con patrón difuso cuando al menos el $50 \%$ del área del parénquima presentaba inflamación intersticial, con menos del $25 \%$ del área con fibrosis intersticial. Todas las biopsias fueron diagnosticadas, discutidas y revisadas por el nefropatólogo y el nefrólogo a cargo. No fueron incluidas las biopsias con otros diagnósticos histopatológicos específicos más NTIA asociada.

Este trabajo fue aprobado por el Consejo Científico y el Comité de Ética de la institución.

La totalidad de la información fue procesada de forma automatizada; se usó el paquete estadístico SPSS, versión 22.0. Se utilizó la técnica estadística de análisis de distribución de frecuencias, para cada una de las variables cualitativas fueron calculadas las frecuencias absolutas y relativas (porcentajes). A las variables cuantitativas (edad, creatinina sérica, proteinuria de 24 horas), se les calculó media y desviación estándar (DE). Se utilizó el test no paramétrico de homogeneidad para probar la hipótesis nula $\mathrm{H}_{0}$ de igualdad de la frecuencia de NTIA y de esta como causa de IRA en los tres periodos en que se dividió el estudio (1988$1998,1999-2008$ y 2008-2018). Para esta prueba de hipótesis se fijó un nivel de significación de $\alpha=0.05$.

\section{Resultados}

De las 2,472 biopsias renales de riñones propios practicadas en el centro en el periodo de 1988 a 2018, (incluidos ambos años extremos), o sea en 31 años, 77 (3.1\%) fueron diagnosticadas como NTIA, de ellas, $51(67 \%)$ eran de mujeres. La edad media de los pacientes fue de 43.1 años ( \pm 15.7), la creatinina media de $6.1 \mathrm{mg} / \mathrm{dl}( \pm 4.18)$ y la proteinuria de 24 horas promedio de $0.89 \mathrm{~g}( \pm 0.74)$. Nueve de los pacientes tenían 60 años y más.

Al estudiar la frecuencia de este diagnóstico por periodos (Fig. 1), se observa que el porcentaje de NTIA respecto al total de biopsias se ha ido incrementando por décadas, así, de 1988 a 1998, el 1.1\% de los pacientes biopsiados presentaron este diagnóstico, de 1999 a 2008, el 3\%, y de 2008 a 2018, el 4.5\% $(p=0.044)$. Asimismo, ha aumentado este diagnóstico entre los pacientes que se presentan con IRA (Fig. 2). Obsérvese que la frecuencia porcentual de NTIA como causa de IRA se ha incrementado notoriamente en la 


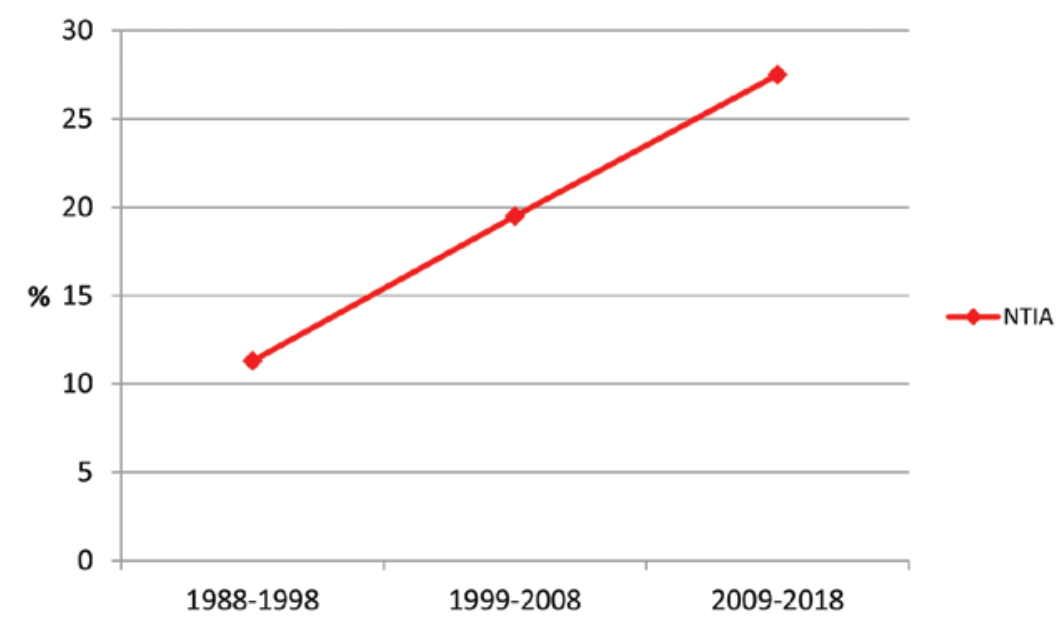

Figura 2. Diagnóstico de NTIA en los pacientes con injuria renal aguda según el período de estudio. NTIA: nefritis tubulointersticial aguda.

última década (27.5\%) con respecto al periodo de 1988 a $1998(11.3 \%)(p=0.04)$.

Al analizar las formas de presentación (Tabla 1) resulta taxativo el predominio de la IRA, en tanto el resto de las formas de presentación en su conjunto no alcanzan el $17 \%$.

La presencia de manifestaciones de respuesta de hipersensibilidad, rash cutáneo, eosinofilia y fiebre, en su conjunto (tríada), solo se halló en el $11.7 \%$ de los pacientes (Tabla 1), y la manifestación más frecuente de forma aislada fue la eosinofilia, que apareció en el $26 \%$ de los casos. El examen del sedimento urinario mostró que $46(74.2 \%)$ pacientes presentaron leucocituria, incluidos $29(46.8 \%)$ que presentaron leucocituria más hematuria, en tanto, el $25.8 \%$ tuvo un sedimento pálido (Tabla 1). Debe tenerse presente que 15 pacientes (19.5\%) presentaban anuria u oliguria extrema al momento de la biopsia, por lo que no se examinó el sedimento urinario.

La causa más común de las NTIA fue la medicamentosa $(79.2 \%)$, seguida por el síndrome de TINU (7.8\%) y el origen autoinmune (3.9\%) (Tabla 1). De los casos de origen autoinmune, dos se debieron a LES y uno a síndrome de Sjögren. Debe tenerse presente que en el $6.5 \%$ de los pacientes no se pudo precisar el origen de la NTIA. Las dos NTIA de origen infeccioso se debieron a leptospirosis.

Los medicamentos que más comúnmente dieron lugar al cuadro fueron el ciprofloxacino y el cotrimoxazol (sulfaprim) que causaron el $42.6 \%$ de los casos, seguidos por las penicilinas, el omeprazol y los antiinflamatorios no esteroideos (AINE) (Tabla 1). Obsérvese que
Tabla 1. Características clínicas y etiología de las nefritis tubulointersticiales agudas estudiadas

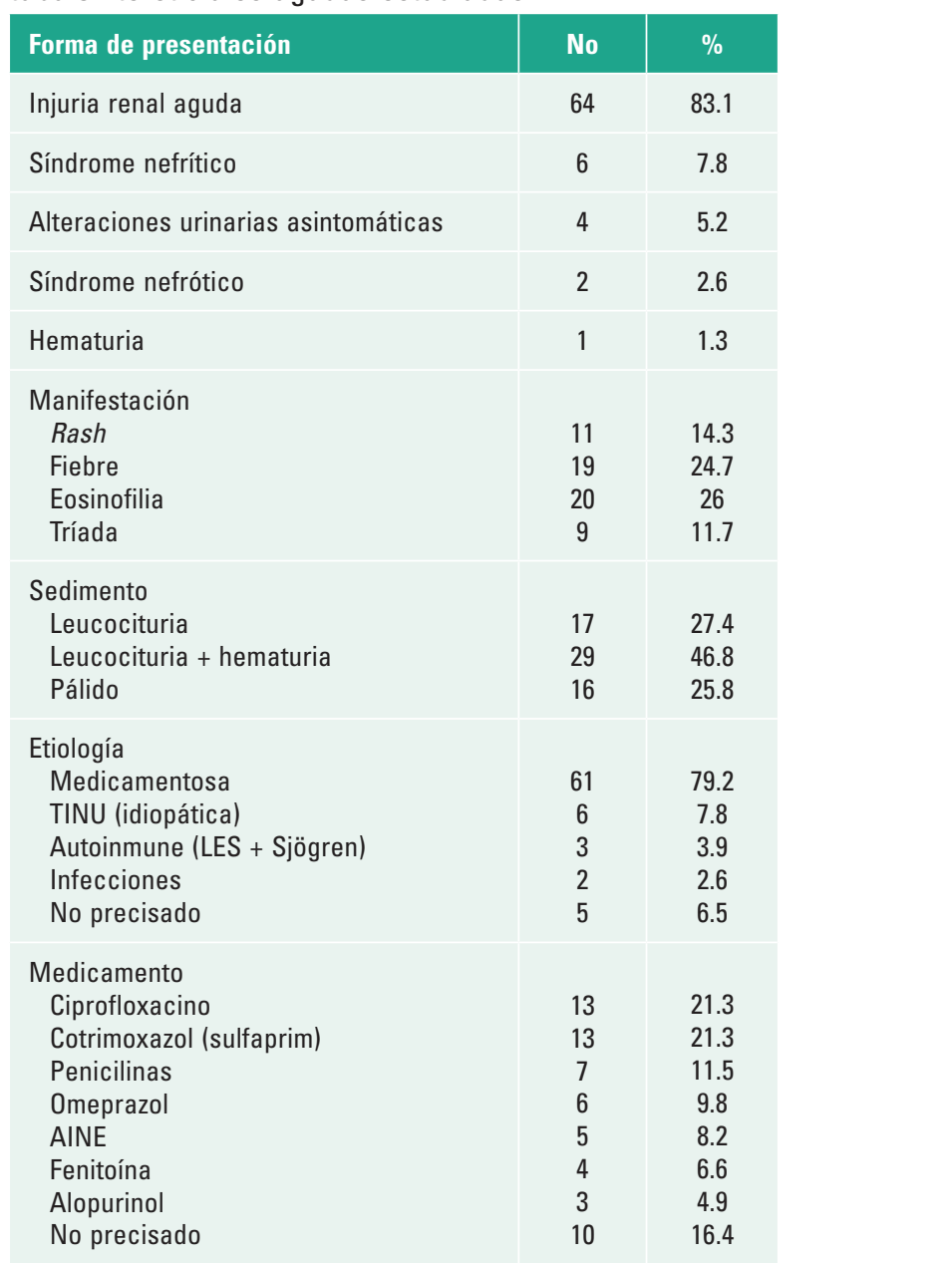

AINE: antiinflamatorios no esteroideos; LES: lupus eritematoso sistémico; TINU: síndrome de nefritis tubulointersticial y uveítis. 


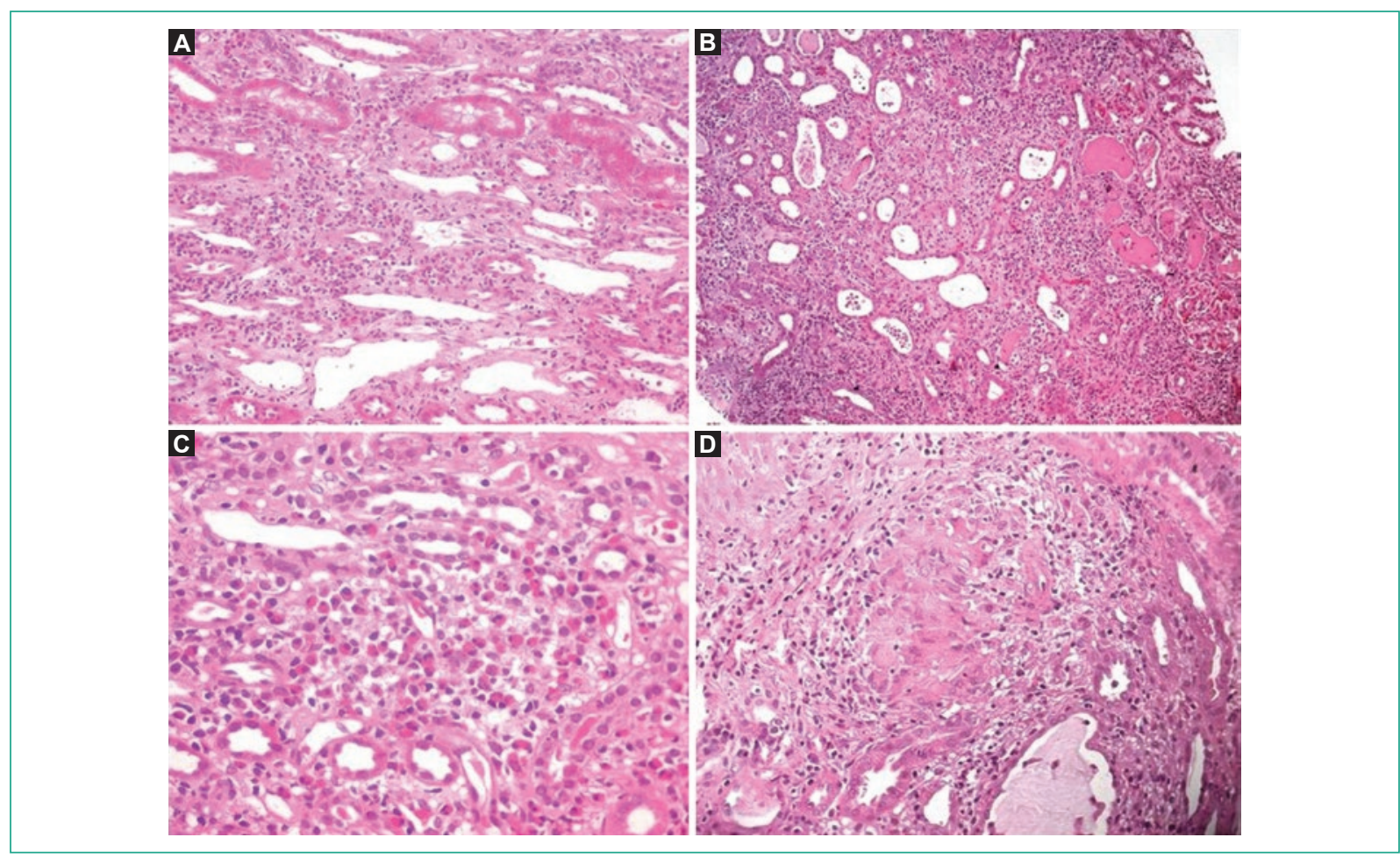

Figura 3. A: nefritis tubulointersticial aguda en paciente con injuria renal aguda, H/E X200; B: infiltrado inflamatorio intersticial difuso con abundantes eosinófilos, hematíes en la luz de los túbulos y áreas de necrosis tubular en paciente con síndrome nefrítico, H/E X200; C: nefritis tubulointersticial aguda con abundantes eosinófilos en paciente con síndrome nefrótico, H/E X400; D: nefritis tubulointerticial granulomatosa en paciente con injuria renal aguda, H/E X400.

los antimicrobianos constituyeron la causa del $54.1 \%$ de las NTIA de origen medicamentoso. Hay que apuntar que no se pudo identificar el medicamento causal en 10 pacientes (16.4\%), pues se encontraban utilizando varios medicamentos al unísono.

Desde el punto de vista histológico solo tres de los pacientes presentaron NTIA granulomatosas (3.9\%), todas de origen medicamentoso, y una de ellas se presentó como parte de un síndrome de DRESS (drug reaction with eosinophilia and systemic symptoms). No se observaron diferencias notorias a la microscopia óptica entre las diferentes formas de presentación, excepto la presencia de hematíes intratubulares en pacientes con hematuria y síndrome nefrítico (Fig. 3).

\section{Discusión}

El aumento en la frecuencia de presentación de NTIA en general y entre los pacientes que se presentan con IRA ha sido identificado en varios estudios, entre los que destaca el estudio del Registro Español de Glomerulonefritis, que incluyó 468 pacientes con NTIA, con una prevalencia total del $2.7 \%$, y del $12.9 \%$ entre los pacientes que se presentaron con IRA ${ }^{4,14,15}$. Las cifras halladas en el presente estudio son algo superiores, especialmente cuando se toman en consideración los resultados de la última década.

Debe tenerse presente que el número de pacientes de 60 años y más en esta serie es escaso, de modo que no consideramos que este segmento poblacional sea el responsable del incremento en la frecuencia de presentación, como se sugiere en otros estudios ${ }^{4,16}$. Consideramos que puede deberse al incremento identificado en la utilización de medicamentos en la población general, y particularmente entre las féminas, que constituyeron dos terceras partes de los $\operatorname{casos}^{17,18}$. El predominio en el sexo femenino ha sido reportado en otras series ${ }^{11,12}$.

Por otra parte, la ausencia de modificaciones en los criterios de biopsia renal en el periodo de estudio hace que los resultados no estén viciados por una mayor tendencia a practicar biopsias, especialmente en pacientes que se presentan con IRA.

El análisis de la forma de presentación denota que, como en todos los estudios revisados, la IRA es la forma predominante, aunque otras presentaciones 
pueden ocurrir y deben tenerse en cuenta a la hora de indicar la realización de una biopsia renal por sospecha de NTIA $4,7,11,12$.

La aparición de signos de respuesta de hipersensibilidad, como rash, fiebre y eosinofilia, forma parte de la descripción clásica de la entidad y ponen alerta al clínico sobre este diagnóstico ${ }^{19}$; $\sin$ embargo, en nuestra serie, como en otros estudios, estos hallazgos fueron menos comunes que en los reportes iniciales, así, la frecuencia de estos signos fue más baja que en dos series retrospectivas que incluyeron 121 pacientes $8,9,11,20$.

El sedimento urinario es una herramienta muy útil para el diagnóstico. La leucocituria, presente en más de las dos terceras partes de los casos, coincide con los hallazgos de otros estudios, aunque en el trabajo del Registro Español de Glomerulonefritis, su presencia es sustancialmente más baja, no obstante, sus autores plantean que esta baja frecuencia de leucocituria pudo deberse a la forma de recolección de los datos $^{4,11,20}$. La frecuencia identificada de oliguria fue inferior a otros reportes ${ }^{9}$.

La magnitud de la proteinuria hallada en esta serie, o sea inferior a $1 \mathrm{~g} / d i ́ a$, resulta semejante a la de otros estudios, y pude tener relación con el escaso número de pacientes ancianos en este trabajo, pues en los adultos mayores con NTIA se han encontrado mayores cifras de proteinuria $11,16,20$.

La frecuencia de las diferentes etiologías de las NTIA suele variar entre los reportes, aunque el origen medicamentoso predomina en casi todos ${ }^{5,12}$. No obstante, hay reportes de India y África donde hasta el $50 \%$ de los casos tienen origen infeccioso $0^{6,21,22}$. El síndrome de TINU tuvo una frecuencia elevada comparada con otros estudios, y destaca que la mayoría de los casos han sido diagnosticados en la última década ${ }^{9,23}$.

Desde la primera descripción de NTIA causada por fármacos hecha por Baldwin en 1968, el listado de medicamentos causantes de NTIA no ha dejado de incrementarse, si bien los antimicrobianos, los AINE y los inhibidores de la bomba de protones suelen ser los más comúnmente identificados ${ }^{12,19,24}$. En este trabajo destaca el amplio predominio de los antimicrobianos frente a los AINE, lo que, si bien se corresponde con otras series, contrasta con algunos estudios que identifican una clara preponderancia de los AINE $^{5,11,12,15}$.

El patrón histológico de infiltración intersticial de las NTIA es habitualmente difuso, aunque una fracción variable, según los diferentes estudios, puede tener una infiltración con formación de granulomas, que si bien puede verse por cualquier etiología, resulta más común en la sarcoidosis y en las de origen infeccioso ${ }^{25}$. Sin embargo, en este estudio, todos los casos con formación de granulomas fueron de origen medicamentoso, uno de ellos con afectación multisistémica ${ }^{26}$.

La ausencia de cambios histológicos distintivos por forma de presentación se corresponde con los hallazgos de estudios similares, aunque en trabajos que han contado con microscopia electrónica se suele encontrar fusión de los pedicelos de los podocitos en pacientes con síndrome nefrótico, especialmente en NTIA causadas por AINE ${ }^{5,9,11,15}$. Además, a esto contribuyó que en esta serie no se incluyeran biopsias de pacientes con otro diagnóstico histopatológico asociado a NTIA.

Este estudio, si bien monocéntrico y sin seguimiento de los pacientes luego de la biopsia renal, incluye un número importante de pacientes, en un lapso de 31 años, sin modificación en los criterios de biopsia, lo que hace que sus resultados sean de utilidad clínica.

\section{Conclusiones}

La NTIA, en este centro de Cuba, predomina en mujeres, su frecuencia se ha incrementado en los últimos tiempos, especialmente entre los pacientes con IRA, que es su forma de presentación más usual. Las manifestaciones de respuesta de hipersensibilidad (rash cutáneo, eosinofilia y fiebre) no fueron comunes, en tanto la leucocituria fue un hallazgo usual en el sedimento urinario. Los medicamentos, especialmente los antimicrobianos, son su causa principal.

\section{Conflicto de intereses}

Los autores declaran no presentar ningún tipo de conflicto de intereses que pueda afectar los resultados y los contenidos en relación con la presente publicación.

\section{Responsabilidades éticas}

Protección de personas y animales. Los autores declaran que para esta investigación no se han realizado experimentos en seres humanos ni en animales.

Confidencialidad de los datos. Los autores declaran que han seguido los protocolos de su centro de trabajo sobre la publicación de datos de pacientes.

Derecho a la privacidad y consentimiento informado. Los autores han obtenido el consentimiento informado de los pacientes y/o sujetos referidos en el 
artículo. Este documento obra en poder del autor de correspondencia.

\section{Bibliografía}

1. Rossert JA, Fischer EA. Acute interstitial nephritis. En: Johnson RJ, Feehally J (Eds.). Comprehensive Clinical Nephrology. Vol. 1. Philadelphia: Elsevier Limited, 2003. p. 769.

2. Cameron JS. Allergic interstitial nephritis: clinical features and pathogenesis. Q J Med. 1998;66:97-115.

3. Petterson E, Von Bonsdorff M, Tornroth T. Nephritis among young Finnish men. Clin Nephrol. 1984;22:217-22.

4. Goicoechea M, Rivera F, López-Gómez JM. Spanish Registry of Glomerulonephritis. Increased prevalence of acute tubulointerstitial nephritis. Nephrol Dial Transplant. 2013;28:112-5.

5. Muriithi AK, Leung N, Valeri AM, Cornell LD, Sethi S, Fidler ME, et al. Biopsy-proven acute interstitial nephritis, 1993-2011: a case series. Am J Kidney Dis. 2014;64:558-66.

6. Praga M, Sevillano A, Auñón P, González E. Changes in the aetiology, clinical presentation and management of acute interstitial nephritis, an increasingly common cause of acute kidney injury. Nephrol Dial Transplant. 2015;30:1472-9.

7. Kodner CM, Kudrimoti A. Diagnosis and management of acute interstitial nephritis. Am Fam Physician. 2003;67:2527-34.

8. Baker RJ, Pusey CD. The changing profile of acute tubulointerstitial nephritis. Nephrol Dial Transplant. 2004:19:8-11.

9. Praga M, González E. Acute interstitial nephritis. Kidney Int. 2010;77: 956-61.

10. Sigala JF, Biava CG, Hulter HN. Red blood cell casts in acute interstitial nephritis. Arch Intern Med. 1978;138:1419-21.

11. Clarkson MR, Giblin L, O'Connell FP. Acute interstitial nephritis: clinical features and response to corticosteroid therapy. Nephrol Dial Transplant. 2004;19:2778-83.

12. Urrestarazú A, Figueroa S, Coitiño E, Coria V, Acosta N, Aunchayna MH, et al. Nefropatías tubulointersticiales no obstructivas biopsiadas en Uruguay. Nefrol Latinoam. 2017;14(4):144-52.
13. Kidney Disease Improving Global Outcomes. KDIGO Clinical practice guideline for glomerulonephritis. Kidnet Int Suppl. 2012;2:1-274.

14. Rychlík I, Jancová E, Tesar V, Kolsky A, Lácha J, Stejskal J, et al. The Czech registry of renal biopsies. Occurrence of renal diseases in the years 1994-2000. Nephrol Dial Transplant. 2004;19:3040-9.

15. Perazella MA, Markowitch GS. Drug-induced acute interstitial nephritis. Nat Rev Nephrol. 2010;6:461-70.

16. Haas M, Spargo BH, Wit EJ, Meehan SM. Etiologies and outcome of acute renal insufficiency in older adults: a renal biopsy study of 259 cases. Am J Kidney Dis. 2000;35:433-47.

17. García AJ, López L, Pría MC, León P. Consumo de medicamentos y condiciones de vida en un municipio de La Habana. Rev Cubana de Salud Pública. 2018;44(1):27-45.

18. García AJ, Alonso L, López P. Consumo de medicamentos en la población femenina adulta de Cuba. Rev Cubana Salud Pública. 2013;39(3):440-9.

19. Baldwin DS, Levine BB, McCluskey RT, Gallo GR. Renal failure and interstitial nephritis due to penicillin and methicillin. N Engl J Med. 1968; 279:1245-52.

20. González E, Gutiérrez E, Galeano C, Chevia C, de Sequera P, Bernis C et al. Early steroid treatment improves the recovery of renal function in patients with drug-induced acute interstitial nephritis. Kidney Int. 2008;73:940-6.

21. Soliman AR. Spectrum of acute kidney injury in a tertiary care hospital in Cairo. Arab J Nephrol Transplant. 2011;4:83-6.

22. Das U, Dakshinamurty KV, Prayaga A. Pattern of biopsy-proven renal disease in a single center of south India: 19 years experience. Indian J Nephrol. 2011;21:250-7.

23. Bacallao Méndez RA, López Marín L, Llerena Ferrer B. Síndrome de nefritis tubulointersticial y uveítis. Rev Cubana Med. 2016;55(4):311-8.

24. Torpey N, Barker T, Ross C. Drug-induced tubulo-interstitial nephritis secondary to proton pump inhibitors: experience from a single UK renal unit. Nephrol Dial Transplant. 2004;19:1441-6.

25. Joss N, Morris S, Young B, Geddes C. Granulomatous interstitial nephritis. Clin J Am Soc Nephrol. 2007;2:222-30.

26. Bacallao Méndez R, López Marín L, Martínez Pérez D. Síndrome de hipersensibilidad a anticonvulsivantes y nefritis túbulointersticial aguda granulomatosa tras tratamiento con fenitoína. Rev. cuban invest. bioméd [Internet]. 2019 [citado 2019 Jul ${ }^{11} ; 37(3)$ :[aprox. 0 p.]. Disponible en: http://www.revibiomedica.sld.cu/index.php/ibi/article/view/139. 